\title{
Far Field Plume Distribution and Divergence for NEXT: DART Mission
}

\author{
J.A. Young ${ }^{1}$, T.S. Matlock, M. Nakles, M.W. Crofton ${ }^{2}$ \\ The Aerospace Corporation, El Segundo, CA 90245, USA \\ M. J. Patterson ${ }^{3}$, N. Arthur \\ NASA Glenn Research Center, Cleveland, Ohio 44135, USA \\ and \\ J. W. John ${ }^{4}$ \\ Johns Hopkins University Applied Physics Laboratory, Laurel, Maryland 20723, USA
}

\begin{abstract}
In support of the Double Asteroid Redirection Test (DART) mission, laboratory measurements were made on the NEXT ion engine, which will be used for the spacecraft's inspace propulsion [1]. This study revisits a small range of mission-specific $2.7 \mathrm{~A}$ throttle levels to understand the effect of in-flight flow rate variability, investigate intermediate throttle conditions, and improve measurement methodology. This paper specifically examines the farfield plume divergence and backflow ion flux distribution of the NEXT, while a companion paper examines the charge state distributions.
\end{abstract}

\section{Nomenclature}

$I_{b} \quad=$ total beam current emitted from the thruster

$I_{Z} \quad=$ component of total beam current parallel to the thrust axis

$\vec{J}=$ current density vector

$J_{\text {probe }}=$ current density measured at a plasma probe face

$J_{\psi} \quad=$ current density measured at distance $r_{0}$ normal to the thruster grid

$J_{\delta} \quad=$ current density measured at distance $r_{0}$ normal to the expected ion trajectory, $\delta$

$r_{0}=$ nominal distance of probe from the center of the thruster grid when $\psi=\theta=0$

$\vec{r}_{\text {probe }}=$ location of probe relative to grid radius of curvature origin

$\vec{R}(\psi) \quad=$ radius of curvature vector terminating at grid at angle $\psi$

$\hat{z}=$ unit vector along the thrust axis

$\beta=$ divergence correction factor

$\theta=$ angle relative to the thruster centerline with origin at the center of the thruster grid

$\psi \quad=$ angle relative to the thruster centerline with origin at the grid radius of curvature

$\zeta \quad=$ angle relative to the thruster centerline with origin at the edge of the grid

$\delta=\quad$ expected angle of ions relative to thruster centerline at location of probe

\section{Introduction}

The performance characteristics of the NEXT (NASA Evolutionary Xenon Thruster) gridded ion engine have been heavily studied over the years and it has undergone extensive lifetime testing [1-9, 18-20]. Recently, it has been selected for use in the Double Asteroid Redirection Test (DART), in which a satellite will demonstrate asteroid deflection by kinetically impacting the smaller asteroid, Didymoon, in a binary asteroid system [10]. This mission has

\footnotetext{
${ }^{1}$ Senior Member of the Technical Staff, Propulsion Science Department, M2-341, jason.a.young@aero.org

${ }^{2}$ Senior Scientist, Propulsion Science Department, M2-341, mark.w.crofton@aero.org

${ }^{3}$ Senior Technologist, Propulsion Division, MS 301-3

${ }^{4}$ Propulsion Lead, DART Mission, Johns Hopkins Applied Physics Laboratory
} 
tight operational requirements, such that the existing NEXT data, although voluminous, is inadequate. Of particular mission interest is the performance within a narrow range of operating conditions, with beam current of $I_{b}=2.7 \mathrm{~A}$. These conditions are circled in red in Table 1, which shows the NEXT Throttle Table 11.1 (TT11.1). The nominal throttle level is TL28.

Table 1 NEXT throttle table 11.1. Throttle levels of interest, from TL29 to ETL2.7B, are circled in red

\begin{tabular}{|c|c|c|c|c|c|c|c|c|c|c|c|c|c|}
\hline \multicolumn{3}{|c|}{ NEXT TT11.1 } & \multicolumn{11}{|c|}{$V_{b p s}, \mathrm{~V}$} \\
\hline$I_{b s} \mathrm{~A}$ & 1800 & 1567 & 1396 & 1179 & 1021 & 936 & 850 & 700 & 679 & 650 & 400 & 300 & 275 \\
\hline 3.52 & TL40 & TL39 & TL38 & TL37 & ETL 3.52A & ETL $3.52 \mathrm{~B}$ & ETL $3.52 \mathrm{C}$ & ETL 3.52D & & & & & \\
\hline 3.10 & TL36 & TL35 & TL34 & TL 33 & ETL $3.1 \mathrm{~A}$ & ETL $3.1 \mathrm{~B}$ & ETL $3.1 \mathrm{C}$ & ETL3.1D & ETL3.1E & & & & \\
\hline 2.70 & TL32 & TL31 & TL 30 & TL29 & TL28 & ETL2.7A & ETL2.7B & ETL2.7C & ETL2.7D & ETL2.7E & & & \\
\hline 2.35 & TL27 & TL26 & TL25 & TL24 & TL23 & ETL $2.35 \mathrm{~A}$ & \begin{tabular}{|l|l} 
ETL $235 \mathrm{~B}$ \\
\end{tabular} & ETL $2.35 \mathrm{C}$ & ETL2.35D & ETL2.35E & & & \\
\hline 2.00 & TL22 & TL21 & TL20 & TL19 & TL18 & ETL2.0A & ETL2.0B & ETL2.0C & ETL2.0D & \begin{tabular}{|l|l|} 
ETL $2.0 \mathrm{E}$ \\
\end{tabular} & & & \\
\hline 1.60 & TL17 & TL16 & TL15 & TL14 & TL13 & ETL1.6A & ETL1.6B & ETL1.6C & ETL1.6D & ETL1.6E & ETL1.6F & & \\
\hline 1.20 & TL12 & TL11 & TL 10 & TL09 & TL08 & TL07 & TL06 & & TL05 & TL04 & TL03 & TL02 & \\
\hline 1.00 & & & & & & & & & & & & & TL01 \\
\hline
\end{tabular}

Specifically, there is an interest in how various thruster characteristics are affected by a modest variation in total discharge xenon flow rate and flow rate to the cathode. The most flooded condition is modified by a suffix of "A," the nominal condition has a suffix of " $\mathrm{H}$ ', and the most lean has a suffix of "O." Hence, the new throttle level DTL28-H$\mathrm{O}$ is DART throttle level 28 with nominal $(\mathrm{H})$ total discharge flow and lean cathode flow (O). The DART test campaign involves multiple measurements of the NEXT, discussed in companion papers [10-13]. This paper concerns only the angular plume profile and divergence.

Angular spreading of the ion engine plume indicates propellant is being accelerated orthogonal to the desired direction of motion. Hence it is a source of thrust inefficiency, typically quantified by a thruster divergence correction factor, beta, defined below:

$$
\beta=\frac{I_{z}}{I_{b}}=\frac{\int \vec{J} \cdot \hat{z} d A}{\int \vec{J} \cdot \hat{n} d A}
$$

Here $\vec{J}$ is the current density at a sufficient distance from the thruster that the angular distribution is unchanging, $\hat{z}$ is the unit vector in the thrust direction, and $\hat{n}$ is the unit vector normal to the integration surface. Ideally, one might expect this distance ought to be very much larger than the radial extent of the thruster, such that the thruster appears as a point-like source [14]. This way, any variations in current density and beam vectoring at the surface of the thruster would be washed out and the far-field density would decay geometrically at $1 / \mathrm{r}^{2}$. According to [15], for a flat gridded ion engine, that distance is greater than eight times the thruster radius. For the NEXT, that would mean probe distances greater than $1.5 \mathrm{~m}$, although one might speculate that its dished grids may alter the calculation.

For experimental purposes, it can be impractical to measure at great distances due to limitations in the dimensions of the chamber and attenuation of primary ions via charge exchange. This is particularly an issue at the walls of the test chamber, where the current density signal may still be strong but motion of a diagnostic probe is constrained. In this paper, it will be shown that with the correct assumptions and correct measurement technique, somewhat shorter distances are adequate to determine far-field plume behavior of dished grids, at least at high angles.

To gain a more comprehensive understanding of the diverging plume from the NEXT ion engine, angular flux distributions were measured at three distances from the thruster grids $(1 \mathrm{~m}, 1.6 \mathrm{~m}$, and 2.8m) using a planar Langmuir probe (PP) and a retarding potential analyzer (RPA) with sufficient bias to reject charge exchange ions. For the $1.6 \mathrm{~m}$ condition alone, the thruster was offset toward the chamber wall to maximize angular range on one side. After performing appropriate geometric corrections $[5,6]$, data collected at $1.0 \mathrm{~m}, 1.6 \mathrm{~m}$ and $2.8 \mathrm{~m}$ distances were found to be self-consistent. This further validates the average ion trajectories proposed in [6], suggests that thruster-wall proximity was not an issue, and suggests that measurements at $1.6 \mathrm{~m}$ alone should be adequate in the future, with even shorter distances, like $1.0 \mathrm{~m}$, possible at least at high angles. It will be shown that divergence and ion flux distribution data at 2.7A throttle levels were in-family with data from previous studies at $2.7 \mathrm{~A}$ and the effect of flow rate was minimal.

\section{Experiment}

The experimental geometry is summarized in Figure 1. The planar probe and retarding potential analyzers were attached to rotating arms at each of the specified distances. The axis of rotation of the $2.8 \mathrm{~m}$ probe arm was the center 
of the thruster grid. The axis of the $1.6 \mathrm{~m}$ probe arm was the radius of curvature of the dished grid (about $71 \mathrm{~cm}$ behind the center of the grid). The axis of the $1.0 \mathrm{~m}$ probe was at the edge of the grids. The thruster was canted by 8 degrees toward the wall to increase the asymmetric angular range of the probes relative to the thruster axis.

Since it was not practical to have all probe arms simultaneously aligned with the thruster, it was necessary to have the thruster move laterally between probe "stations" located at different distances from the chamber wall. The $1.0 \mathrm{~m}$ and $2.8 \mathrm{~m}$ probe rotation stages were at the center of the chamber (relative to the side wall). The $1.6 \mathrm{~m}$ probe arm rotation stage was placed on a lateral motion stage, similar to the thruster. In this way, both $1.6 \mathrm{~m}$ probe axis and thruster could be translated together, varying their distance to the chamber wall. This extra degree of freedom was designed to address a question about whether proximity to the wall could affect the measured plume profile. For most measurements, the probe axis was parked next to the wall to provide the maximal angular range relative to zero.

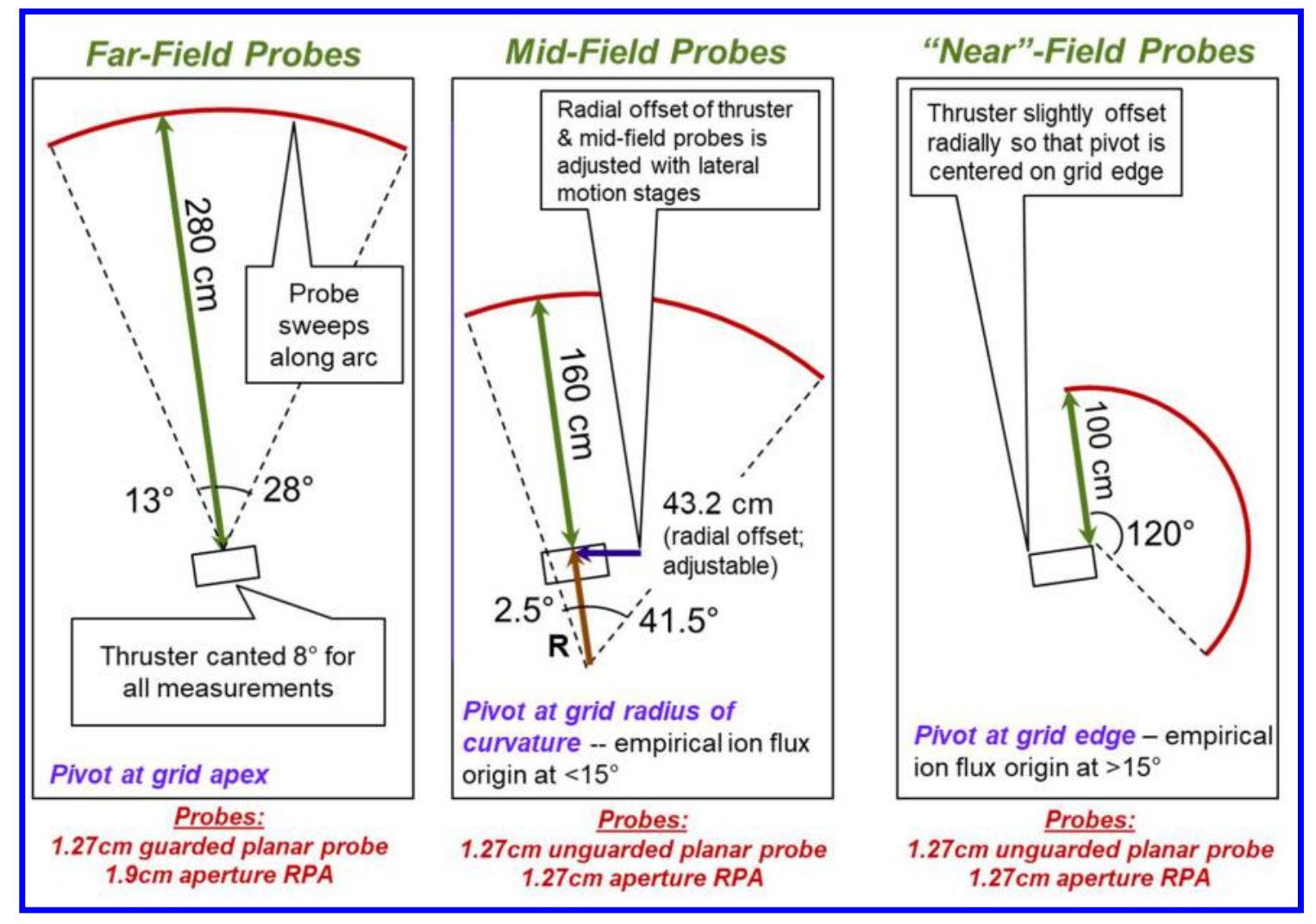

Figure 1 Summary of setup geometry for Far-field (2.8m), Mid-field (1.6m) and "Near"-field probes.

The design of the planar probes was similar to that used in previous studies [5,6]. An unguarded $1.27 \mathrm{~cm}$ diameter planar probe was used to measure absolute current density at $1.0 \mathrm{~m}$ and $1.6 \mathrm{~m}$. A $1.27 \mathrm{~cm}$ guarded planar probe was used at $2.8 \mathrm{~m}$. Unfortunately, the $2.8 \mathrm{~m}$ planar probe developed a guard short early in the measurement campaign so the results were deemed unreliable (hence only relative current densities based on RPA signals are available at $2.8 \mathrm{~m}$ ). The planar probes were biased at $-25 \mathrm{~V}$ then $-20 \mathrm{~V}$ at each angular position using a Keithley 2410 Sourcemeter. At this bias, the probe was presumed to be in ion saturation mode.

The planar probe current includes contributions from charge exchange ions, which do not contribute momentum. In order to determine the fraction of energetic ions at a given plume angle, an RPA was used. The design of the RPA was similar to that used in previous studies [6]. The RPA consisted of square collector inside of two nested boxes with mesh-covered apertures. The aperture was $1.27 \mathrm{~cm}$ for the $1.0 \mathrm{~m}$ and $1.6 \mathrm{~m} \mathrm{RPAs}$ and $1.9 \mathrm{~cm}$ for the $2.8 \mathrm{~m} \mathrm{RPA}$. The outer mesh electrode was biased to $-20 \mathrm{~V}$ or more to reject electrons. The inner mesh was coupled to the collector as this was found to produce the expected flat energy dependence for low power gridded ion sources. The collector was biased at $30 \mathrm{~V}$ then $35 \mathrm{~V}$ and the current collected on a Keithley 2410 Sourcemeter. 


\section{Results}

\section{A. Geometric Corrections}

Absolute current density was determined from the planar probe data around the center of the plume, where charge exchange contributions are expected to be negligible. This was then plotted against RPA current in the same region and the slope was used to renormalize the RPA data. When necessary, an ad hoc angular offset was applied to ensure the peaks for the planar probe and RPA aligned and were centered at $0^{\circ}$. The renormalized RPA data then represented the energetic ion current density at all angles. However, that current density is still expected to depend on the angle of incidence between the ions and the probe face. The expected trajectory of ions emanating within the $\pm 15^{\circ}$ span of the thruster's dished grid is normal to the grid face [6]. In other words, the vector origin is the radius of curvature of the grid. The $1.6 \mathrm{~m}$ probe conforms to this geometry so no correction is required to the measured current density within $\pm 15^{\circ}$. The $2.8 \mathrm{~m}$ probe does not, so a simple cosine correction was required. The $2.8 \mathrm{~m}$ probe also varied in distance from the grids so a $1 / \mathrm{r}^{2}$ distance scaling correction was assumed:

$$
J_{\psi}=\left(\frac{J_{\text {probe }}}{\cos (\theta-\psi)}\right)\left(\frac{r_{0}}{\left|\vec{r}_{\text {probe }}(\theta)-\vec{R}(\psi)\right|}\right)^{2}
$$

Here $\vec{r}_{\text {probe }}(\theta)$ is the position of the probe for given probe angle $\theta$ and $\vec{R}(\psi)$ is the position on the grid surface for grid arc angle $\psi$ (see Figure 2). For convenience, $r_{0}$ is set to $1.6 \mathrm{~m}$ so that all corrected probe measurements can be compared at a common distance

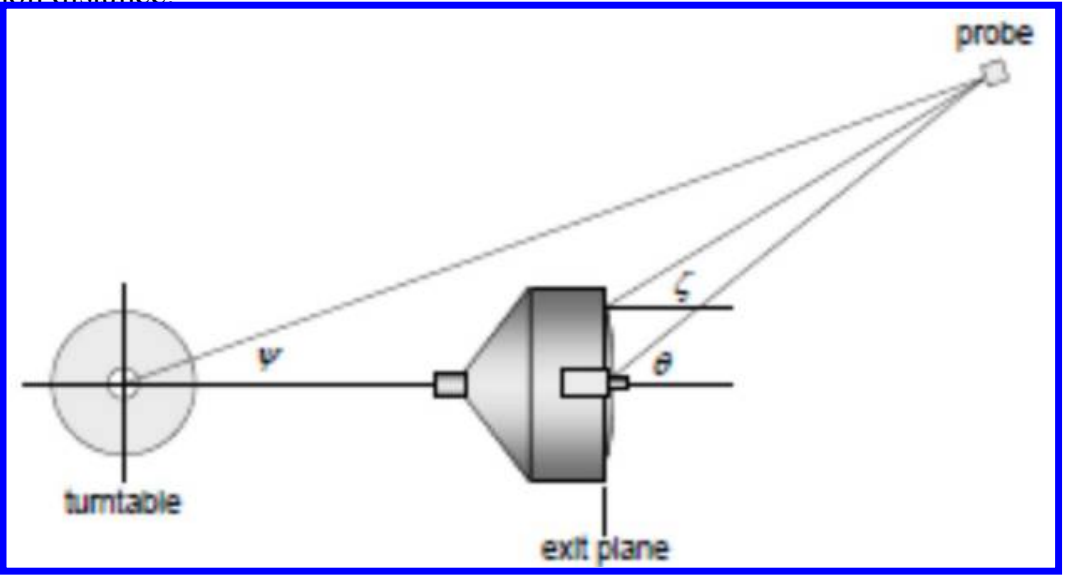

Figure 2 Diagram of the relevant probe angles (from [6]).

Figure 3 shows an example of $1.6 \mathrm{~m}$ and $2.8 \mathrm{~m}$ RPA current density distributions, after the above correction, for the DTL28-H-O throttle condition. The $1.6 \mathrm{~m}$ data shown here were collected with the rotation axis and thruster close to the wall to achieve maximal angular range. To simplify comparison, the $2.8 \mathrm{~m}$ data have been renormalized to the absolute current density at $1.6 \mathrm{~m}$ and $0^{\circ}$. As one can see, converting to grid-normal significantly reduces the apparent spread of the beam and results in a fairly tight match between $1.6 \mathrm{~m}$ and $2.8 \mathrm{~m}$ shape functions. 


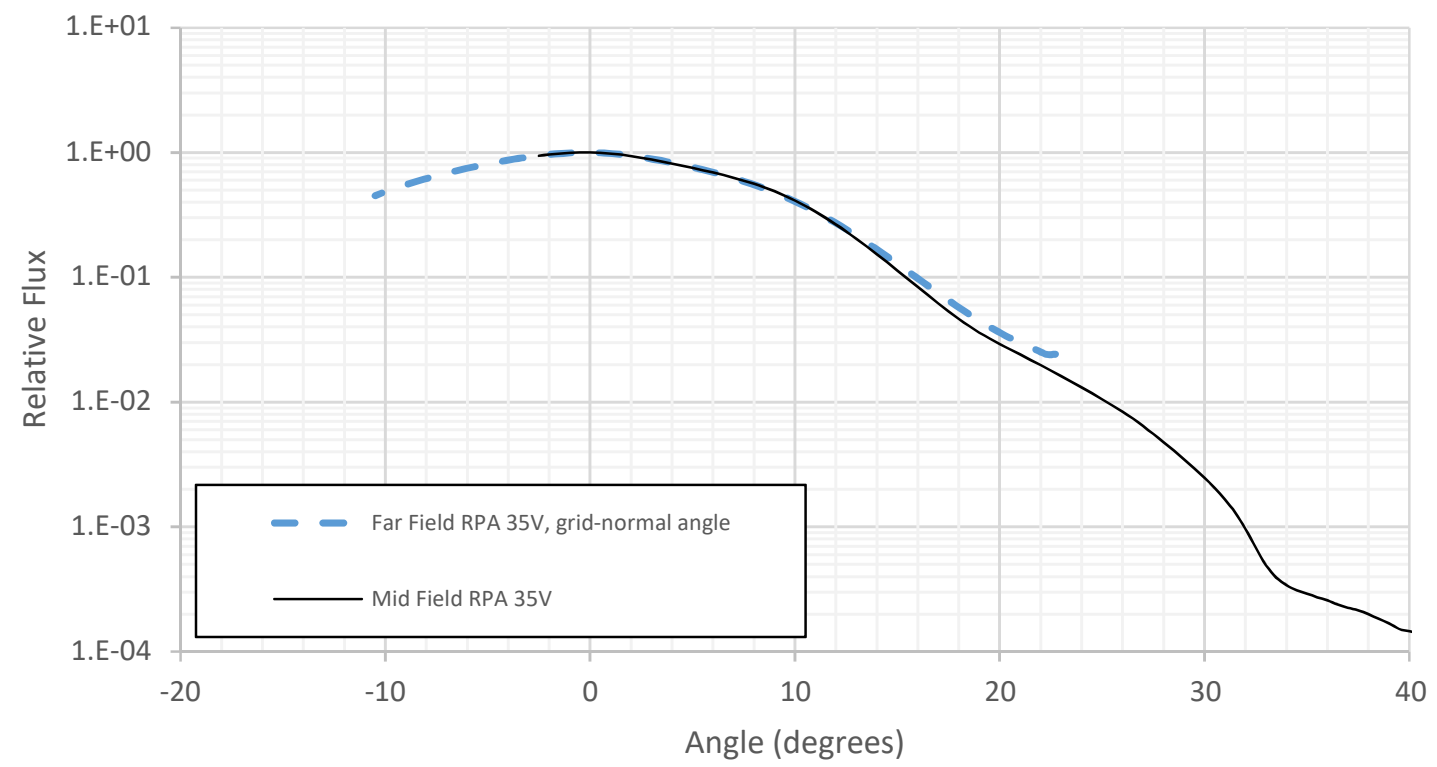

Figure 3 Relative current density for 2.8m (Far Field) and 1.6m (Mid Field) RPAs at DTL28-H-O.

Figure 4 shows a comparison of planar probe and RPA current density measurements for the same DETL2.7B-A$\mathrm{O}$ throttle condition. Here, the diagnostic value of the RPA becomes clear. Beyond $18^{\circ}$, planar probe diverges from the RPA data, becoming nearly flat after $34^{\circ}$ while the RPA continues to drop. At $40^{\circ}$, the charge exchange flux is over an order of magnitude larger than the energetic flux. If this low energy flux were included in divergence factor calculations, it could result in a nontrivial underestimate, particularly if the curve were extrapolated to $90^{\circ}$. The $2.8 \mathrm{~m}$ and $1.6 \mathrm{~m}$ data also diverge slightly at $18^{\circ}$. This is because the grid-normal coordinate system does not accurately describe ion trajectories beyond the angular span of the grid [6]. Also shown in Figure 4, is a comparison of data taken at two lateral offsets of the thruster and probe axis, $x=-17$ (nominal, close to wall) and $x=-7$ (closer to chamber centerline). As one can see, there is negligible difference between the two datasets, validating the decision to move the thruster towards the wall for greater angular range.

Beyond $\pm 15^{\circ}$, Pollard posited that the trajectory should have origin at the edge of the perforated grid, at $\pm 15^{\circ}[6]$. This conclusion was based both on some exploratory measurements and the intuition that at high angles, ions scattered from the outermost beamlet aperture should dominate the signal. In this study, we can examine this assumption more rigorously. Hence, we adopt the piecewise "Pollard" angle $\delta[6]$ using angles defined in Figure 2:

$$
\delta=\psi \quad \text { if }-15^{0}<\psi<-15^{0}, \text { otherwise } \delta=\zeta
$$

The current density correction for the $2.8 \mathrm{~m}$ probe is then slightly modified:

$$
J_{\delta}=\left(\frac{J_{\text {probe }}}{\cos (\theta-\delta)}\right)\left(\frac{r_{0}}{\left|\vec{r}_{\text {probe }}(\theta)-\vec{R}(\delta)\right|}\right)^{2}
$$

where $\vec{R}(\delta)=\vec{R}(\psi)$ if $-15^{0}<\psi<-15^{0}$, otherwise $\vec{R}(\delta)=\vec{R}\left( \pm 15^{0}\right)$

Using these corrections, one can get an excellent match between data at all the distances tested, in the regions where their angles overlap. As shown in Figure 5, any difference between the $1.6 \mathrm{~m}$ and $2.8 \mathrm{~m}$ current density data practically disappears in this coordinate system. The greatest discrepancy is $\sim 16 \%$ and occurs at $15^{\circ}$. This suggests that the true average ion trajectory may transition from grid normal to grid edge coordinates slightly more gradually than a piecewise function. 


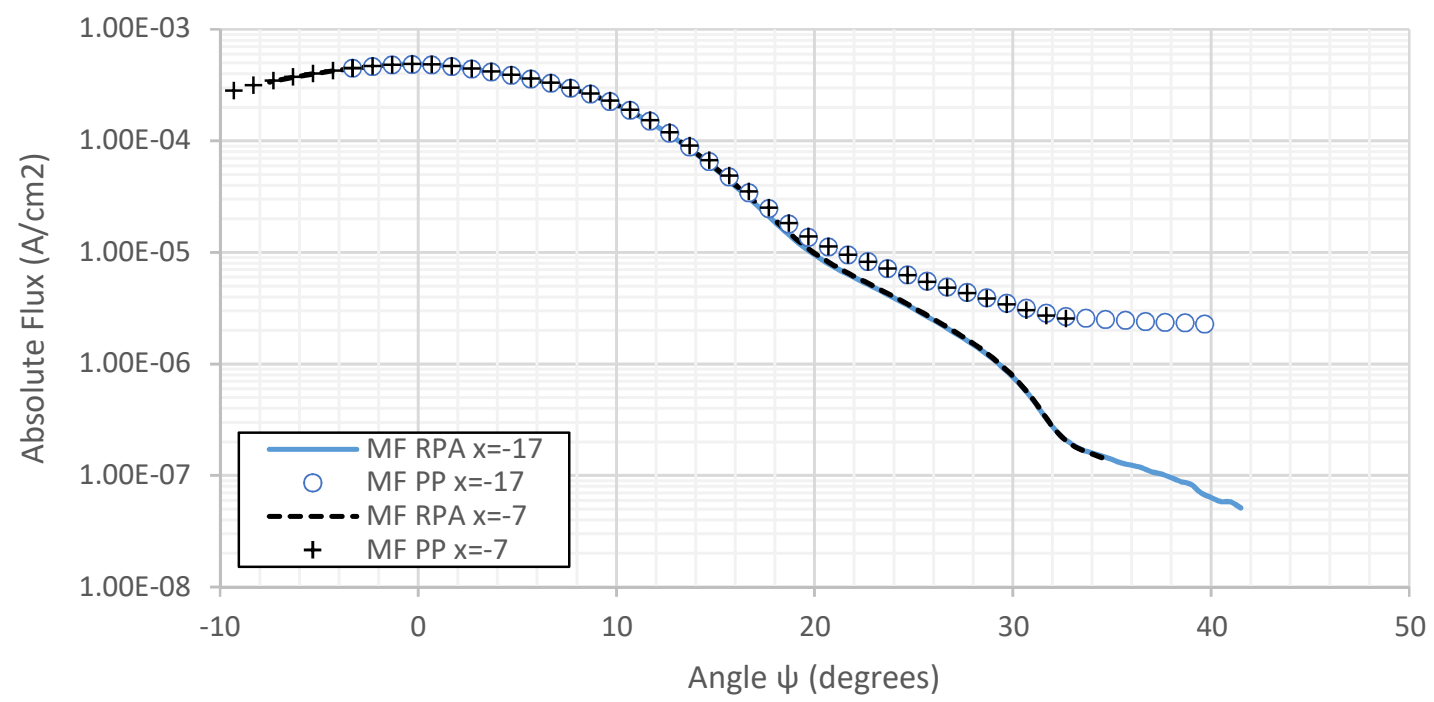

Figure 4 Absolute current densities for 1.6m (MF) RPA and Planar Probes at DETL2.7B-A-O.

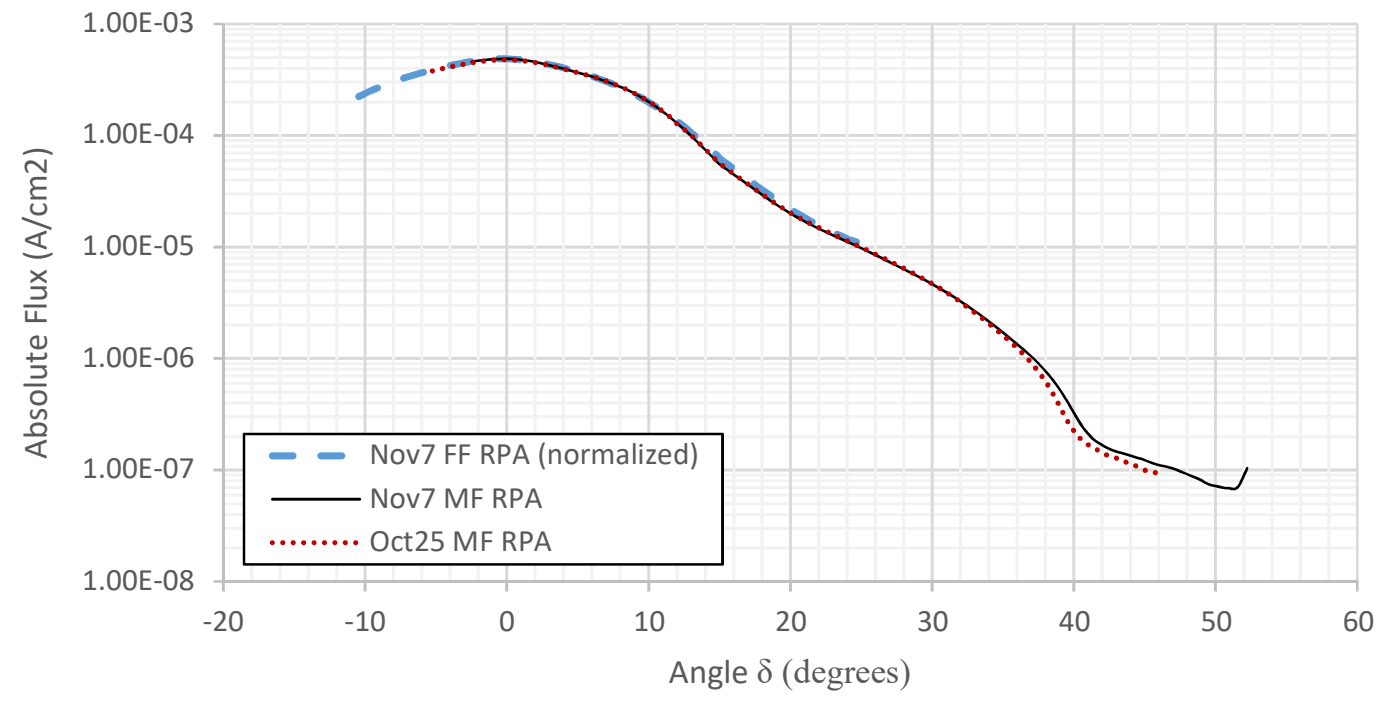

Figure 5 Absolute current density for renormalized 2.8m (FF) and 1.6m (MF) RPAs using transformed angle coordinates $\delta$ at DTL28-H-O.

\section{B. 1.6m Angular Distributions}

Having established in the previous section that the far-field $(2.8 \mathrm{~m})$ and mid-field $(1.6 \mathrm{~m})$ data are functionally identical, it is only necessary to present the $1.6 \mathrm{~m}$ data. Figure 6 shows $1.6 \mathrm{~m}$ current density distributions for all of the throttle conditions tested. In each case, the corresponding curve at $2.8 \mathrm{~m}$ (not shown) was found to be an excellent match. For simplicity, grid normal coordinates are used in these plots. As one can see, there is negligible difference between the flux distributions taken at high $(\mathrm{A})$ nominal $(\mathrm{H})$ and low $(\mathrm{O})$ total discharge flow rates. This is consistent with early measurements of two-grid ion thrusters [16]. In fact, since the range of thruster beam voltages tests was limited and the beam current was fixed, the differences between flux distributions is fairly small, as well. Most of the discrepancy seems to occur beyond $\psi=32^{\circ}$, where instrumental uncertainty is expected to be higher due to low signal 
and electron leakage currents in the RPA. For instance, as shown in Figure 5, data for DTL28-H-O taken on two different days ("Oct 25" and "Nov 7") show some discrepancies. At these higher angles, the data taken at $1 \mathrm{~m}$ appears more reliable for showing qualitative trends, as will be discussed in the next section.

The decay in current density with angle is not a uniform exponential or gaussian, as is typically used in some extrapolations (e.g. [6,17]). Instead, it has a series of inflections or "knees" at different angles. The first knee is around $15^{\circ}$, where the beam transitions to off-grid scaling, which appears slower than the scaling just within the edge of the grids. This is partly an artifact of the piecewise transition in coordinates. The next transition occurs around $30^{\circ}$, where the current density starts dropping faster than exponential with angle. Between $\psi=32^{\circ}$ and $34^{\circ}$, there seems to be another knee where fall-off flattens out (or $\zeta=40^{\circ}$ in the more physically relevant grid edge coordinates). It is possible this simply represents the natural profile of the beamlets convolved over the $15^{\circ} \mathrm{span}$ of the grid. In this scenario, the beamlets from the edge of the grid at $\psi=15^{\circ}$ provide the final cutoff at $\zeta=40^{\circ}$, suggesting a sharp beamlet cutoff at $25^{\circ}$ off normal. This is not to be confused with the beamlet half width at half maximum, which is much smaller.

Included in Figure 6 are five intermediate throttle conditions, "DTLNEW-H-O-X-Y" with beam voltages "X" and accel voltages "Y." These new conditions sit between throttle conditions DTL28 and DTL29. Their curves not surprisingly have similar appearance to the adjacent throttle conditions. Interestingly, the knee feature at high angle seems to move around with different voltage parameters, ruling out the possibility that it is fixed by the geometry of the grids alone. In the context of the conjecture discussed above, this would correspond to changing shapes of the beamlets with voltage parameters.
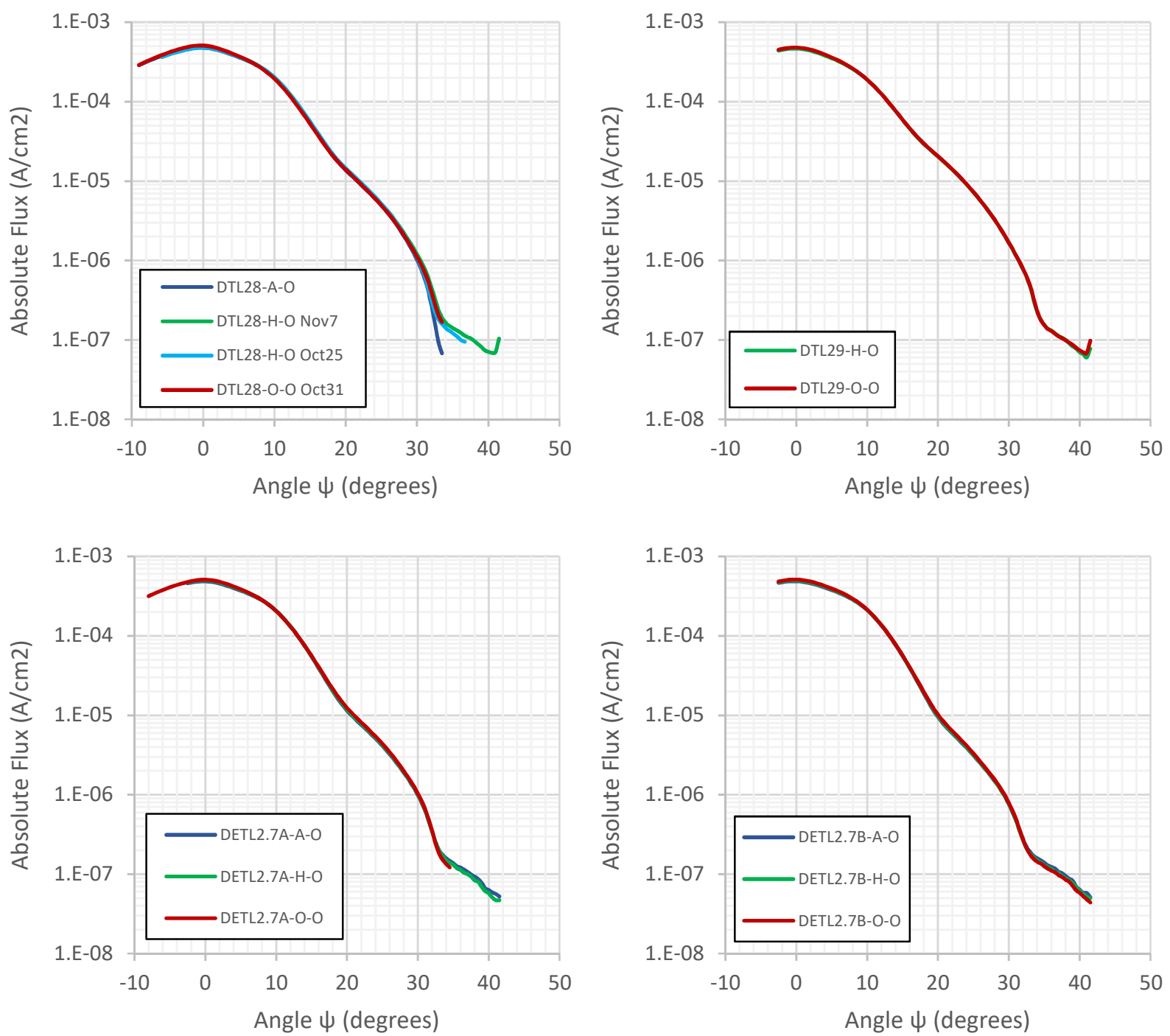


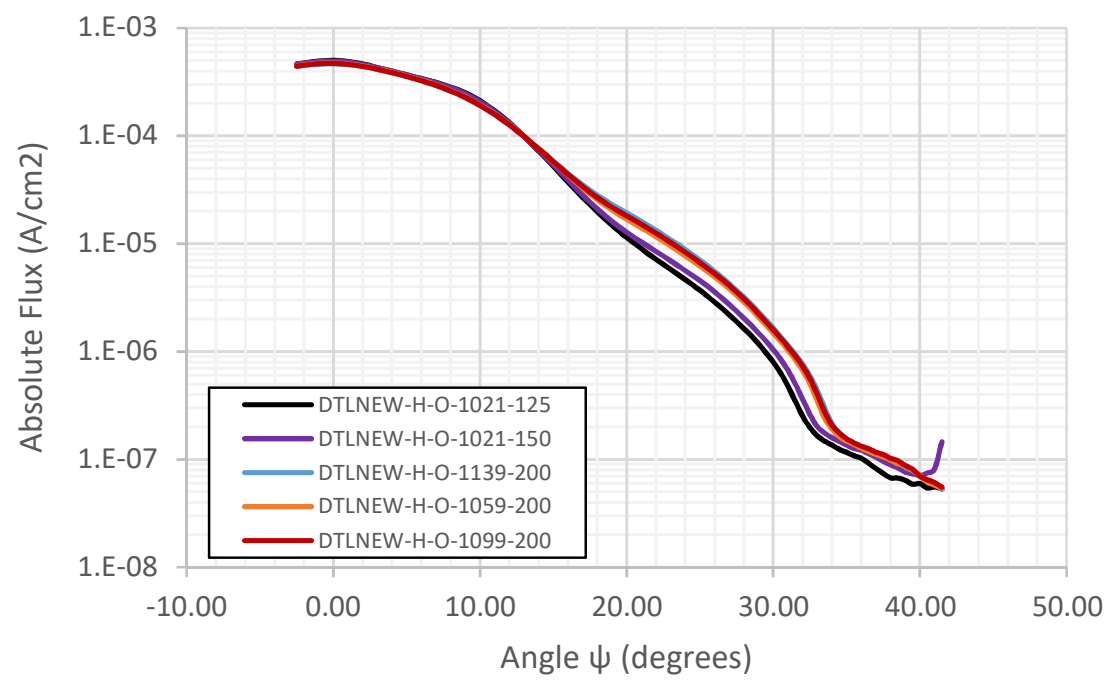

Figure 6 Absolute current density distributions at $1.6 \mathrm{~m}$ for various throttle levels.

\section{1.0m Angular Distributions}

At $1 \mathrm{~m}$, grid-edge coordinates $(\zeta)$ are clearly preferred. As with the $1.6 \mathrm{~m}$ data, both RPA and PP data were recorded. Figure 7 shows a plot of PP and RPA $1 \mathrm{~m}$ current densities collected between $\zeta=0^{\circ}$ and $\zeta=120^{\circ}$ at DTL28-H-O (note that the sign is flipped in the charts). Note that for simplicity, no flux angle or distance correction has been applied to data at $\zeta<15^{\circ}$. For comparison, the corresponding rescaled $1.6 \mathrm{~m} \mathrm{RPA}$ data is plotted as well. Beyond $\zeta=80^{\circ}$, the RPA occasionally indicates non-physical negative current density values. This is attributed to low level electron leakage current. To eliminate these non-physical values and provide a conservative upper bound for high angle flux, the baseline is simply shifted upward such that the most negative value becomes zero. For all intents and purposes, the probe is essentially at its noise floor here so the effective uncertainty should be inferred to be $\sim 10^{-5} \mathrm{~mA} / \mathrm{cm}^{2}$.

The planar probe current density basically remains flat, at around $1-3 \mathrm{uA} / \mathrm{cm} 2$ after $\zeta=50^{\circ}$. Essentially the charge exchange and ambient ions form a diffuse, nearly uniform cloud around the thruster at $1 \mathrm{~m}$. The energetic ion flux drops off precipitously with angle, reaching a noise floor slightly past $\zeta=80^{\circ}$. The $1 \mathrm{~m}$ data closely matches the $1.6 \mathrm{~m}$ data between $\zeta=15^{\circ}$ and $\zeta=40^{\circ}$. The high angle knee observed in the $1.6 \mathrm{~m}$ appears to be replicated in 1.0 data, although possibly shifted closer to $45^{\circ}$. There also appears to be further features, such as an inflection at $55^{\circ}$. The noise floor past $80^{\circ}$ is consistent with the geometrical obstruction of energetic ions by the front mask.

Figure 8 shows a comparison of $1 \mathrm{~m}$ planar probe data for all the thruster throttle levels tested. As one can see, there is little distinction between the conditions and hence no discernible trend with total discharge flow rate. The RPA data, shown in Figure 9 are also very similar for all throttle levels (keeping in mind the noise floor at $\sim 10^{-5}$ $\mathrm{mA} / \mathrm{cm}^{2}$ ). It should be noted that the RPA only rejects ions with energy less than $35 \mathrm{eV}$, so although the ions it collects are energetic, they likely have significantly less energy than beam ions in the center of the plume. In fact, some of these high angle features may be due largely to scattered ions. Hence, if one is concerned with ions with sufficient energy to sputter surfaces, the curves shown here represent an upper bound, with the actual current density of "more" energetic ions likely being much lower. 


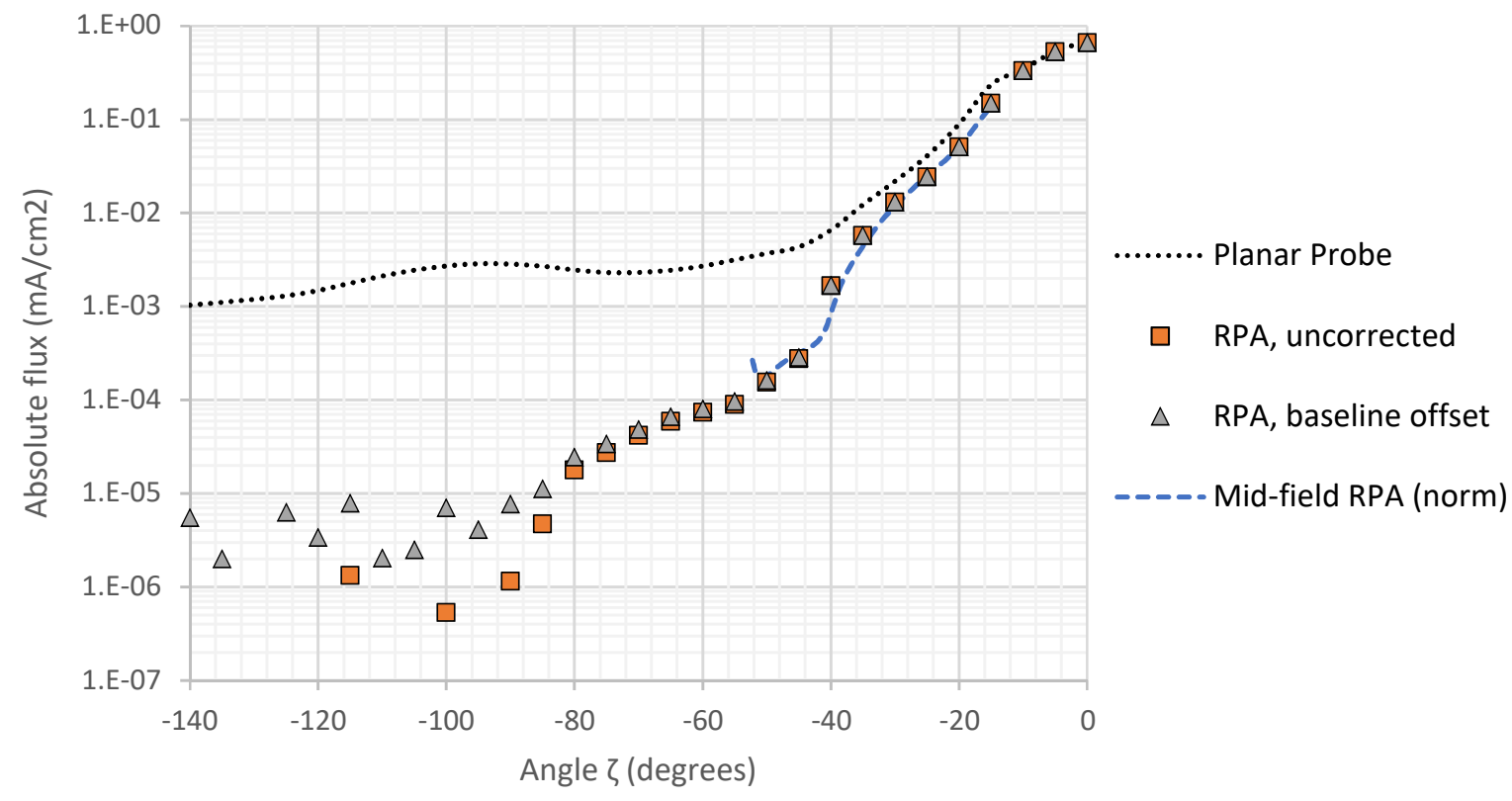

Figure 7 Absolute current density distributions from planar probe and RPA at 1.0m for DTL28-H-O.
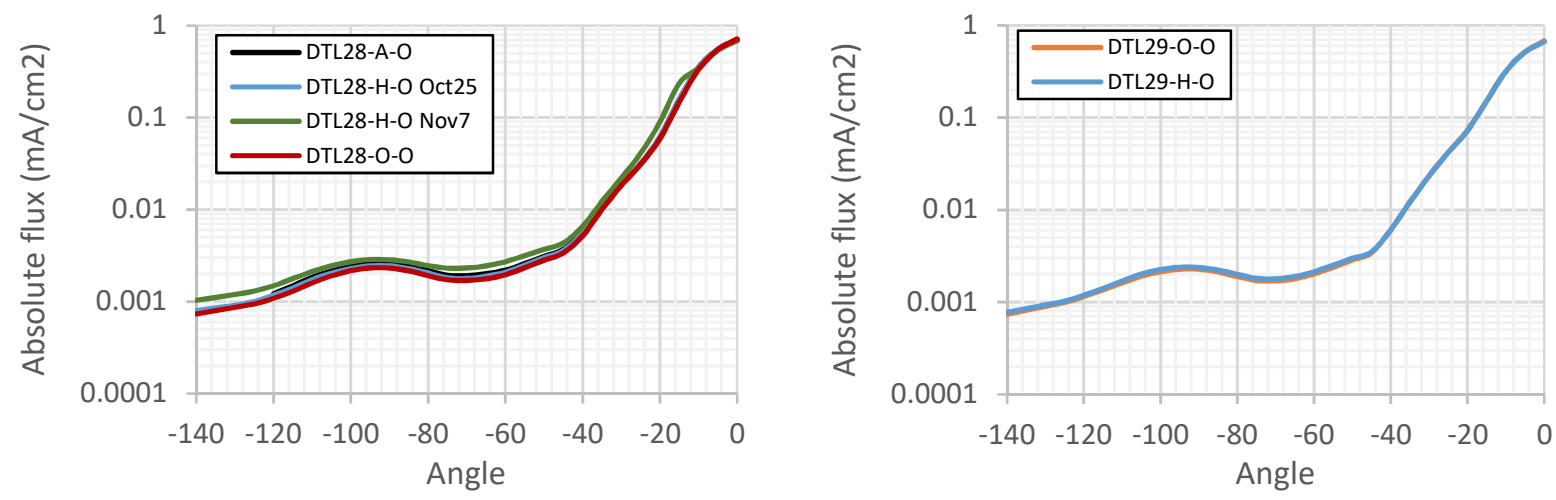

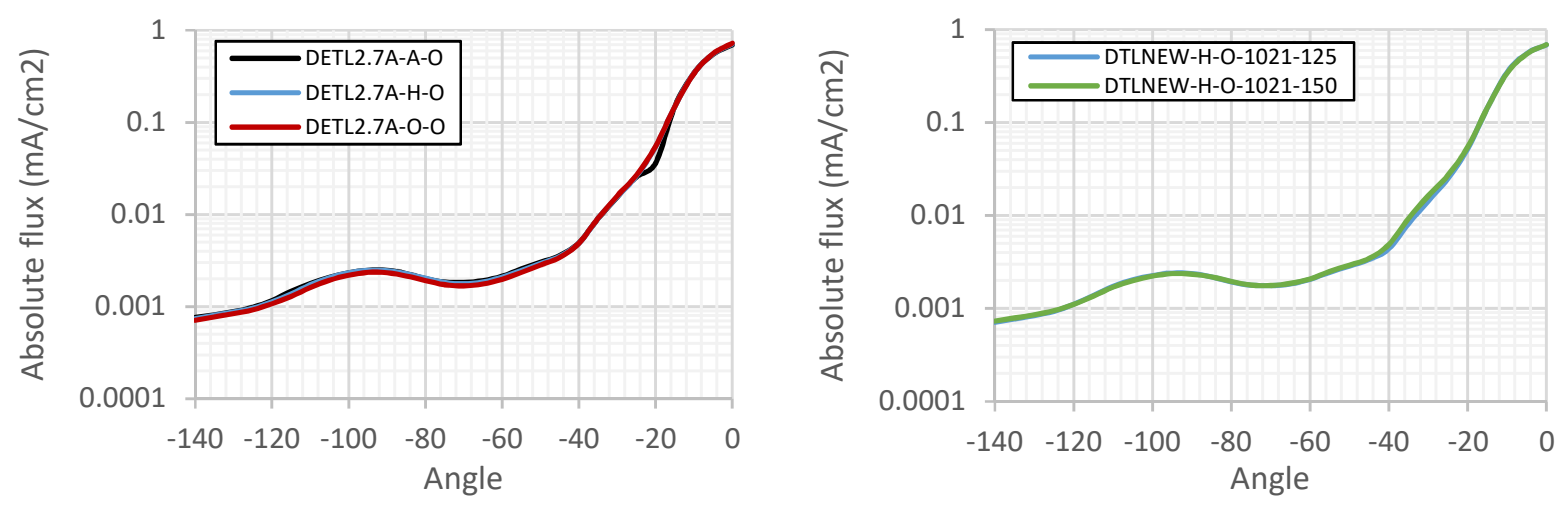

Figure 8 Absolute current density distributions from planar probe at $1.0 \mathrm{~m}$ for various throttle levels.
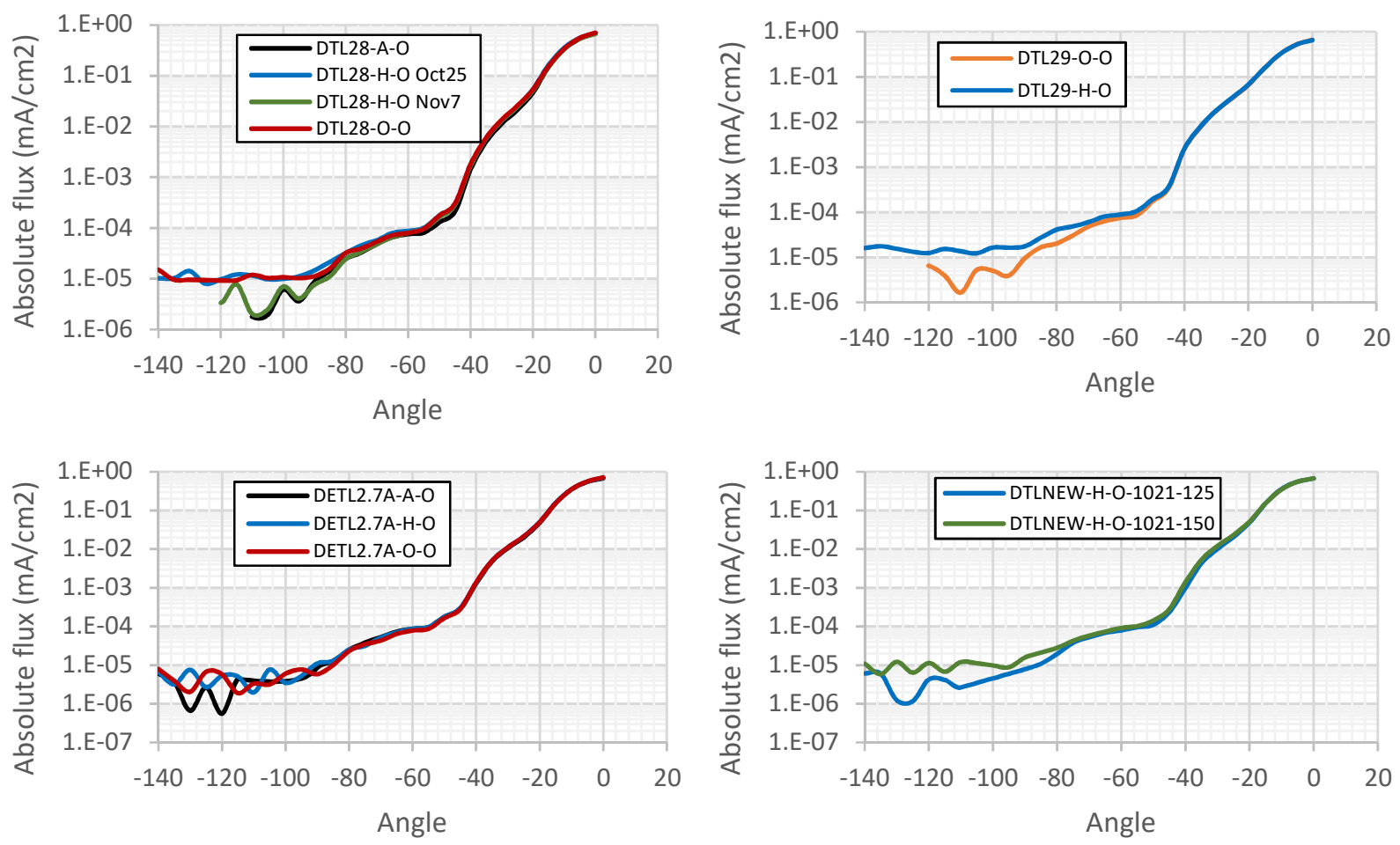

Figure 9 Absolute current density distributions from RPA at 1.0m for various throttle levels.

\section{Divergence Calculation}

As discussed in the introduction, the divergence can be calculated from the angular distribution of energetic current densities. For convenience, the integration is performed over the grid-normal sphere enclosing the thruster at $1.6 \mathrm{~m}$ :

$$
\beta=\frac{I_{z}}{I_{b}}=\frac{2 \pi\left(r_{0}+R\right)^{2} \int_{0}^{\pi} J_{\delta}(\psi) \cos \delta \sin \psi d \psi}{2 \pi\left(r_{0}+R\right)^{2} \int_{0}^{\pi} J_{\psi}(\psi) \sin \psi d \psi}
$$

Note that the function $J_{\psi}(\psi)$ is essentially the current density measured by the grid-normal $1.6 \mathrm{~m}$ probe without any geometric correction. Since the current density measurement at $1.6 \mathrm{~m}$ does not extend beyond $40^{\circ}$, it is necessary to extrapolate the integration, which results in uncertainty. For this study, worst-case uncertainties were assumed. The upper bound for $\beta$ occurred when both the current density and the axial current density remain constant beyond $40^{\circ}$. The lower bound for $\beta$ occurred when the axial current density is zero and only the current density remains constant beyond $40^{\circ}$. The nominal value ignored the extrapolation. In principle, one could argue for smaller uncertainties from 
extrapolation, particularly using insight from the $1.0 \mathrm{~m}$ data. However, there are various other difficult to quantify instrumental effects and assumptions that would grow the overall uncertainty. For instance, we assume a constant distribution and axisymmetry, which is not strictly valid, as discussed in a companion paper on thrust vector measurements [11]. Our uncertainty is also consistent with the empirical scale of discrepancies between plume-derived thrust and actual measured thrust determined in a previous study of the NEXT [7].

Table 2 Divergence factor calculations and predictions (TT 11.1) for all throttle levels tested summarizes the divergence factors for all the throttle levels tested. Figure 10 shows measured data versus beam power supply voltage. Overall, the differences between divergence factors with total discharge flow rate are negligible (parts per thousand), with no discernible trend. Divergence does decrease slightly with increasing beam voltage, closely hewing to empirical predictions based on previous measurements of NEXT within Throttle Table 11.1. Figure 11 plots actual $\beta$ versus predicted, indicating discrepancies of a few parts per 1000, or less than a milliNewton. Comparison with 2010 data for NEXT (taken from a different thruster) is also favorable, agreeing within the error bars.

Table 2 Divergence factor calculations and predictions (TT 11.1) for all throttle levels tested

\begin{tabular}{|c|c|c|c|c|c|}
\hline Throttle condition & $V_{b}$ & $\beta_{\text {nom }}$ & $\beta_{\min }$ & $\beta_{\max }$ & $\beta[T T$ 11.1] \\
\hline DETL2.7B-A-O & 850 & 0.980 & 0.978 & 0.982 & \\
\hline DETL2.7B-H-O & 850 & 0.981 & 0.978 & 0.982 & 0.9776 \\
\hline DETL2.7B-O-O & 850 & 0.981 & 0.979 & 0.982 & \\
\hline DETL2.7A-A-O & 936 & 0.979 & 0.977 & 0.981 & \\
\hline DETL2.7A-H-O & 936 & 0.979 & 0.977 & 0.981 & 0.9773 \\
\hline DETL2.7A-O-O & 936 & 0.979 & 0.973 & 0.981 & \\
\hline DTL28-A-O & 1022 & 0.979 & 0.975 & 0.980 & \\
\hline DTL28-H-O Oct25 & 1022 & 0.978 & 0.973 & 0.979 & 0.9743 \\
\hline DTL28-H-O Nov7 & 1022 & 0.977 & 0.972 & 0.979 & 0.9743 \\
\hline DTL28-0-0-Oct31 & 1022 & 0.977 & 0.969 & 0.980 & \\
\hline TL28 (Pollard 2010) & 1021 & 0.974 & 0.972 & 0.977 & \\
\hline DTL29-H-O & 1180 & 0.974 & 0.971 & 0.976 & 0.9696 \\
\hline DTL29-0-O & 1180 & 0.974 & 0.970 & 0.976 & \\
\hline TL29 (Pollard 2010) & 1179 & 0.970 & 0.966 & 0.973 & \\
\hline DTLNEW-H-O-1139-200 & 1140 & 0.975 & 0.973 & 0.976 & \\
\hline DTLNEW-H-O-1021-150 & 1021 & 0.977 & 0.970 & 0.980 & \\
\hline DTLNEW-H-O-1021-125 & 1021 & 0.980 & 0.978 & 0.981 & \\
\hline DTLNEW-H-O-1099-200 & 1099 & 0.976 & 0.973 & 0.977 & \\
\hline DTLNEW-H-O-1059-200 & 1059 & 0.976 & 0.974 & 0.978 & \\
\hline
\end{tabular}




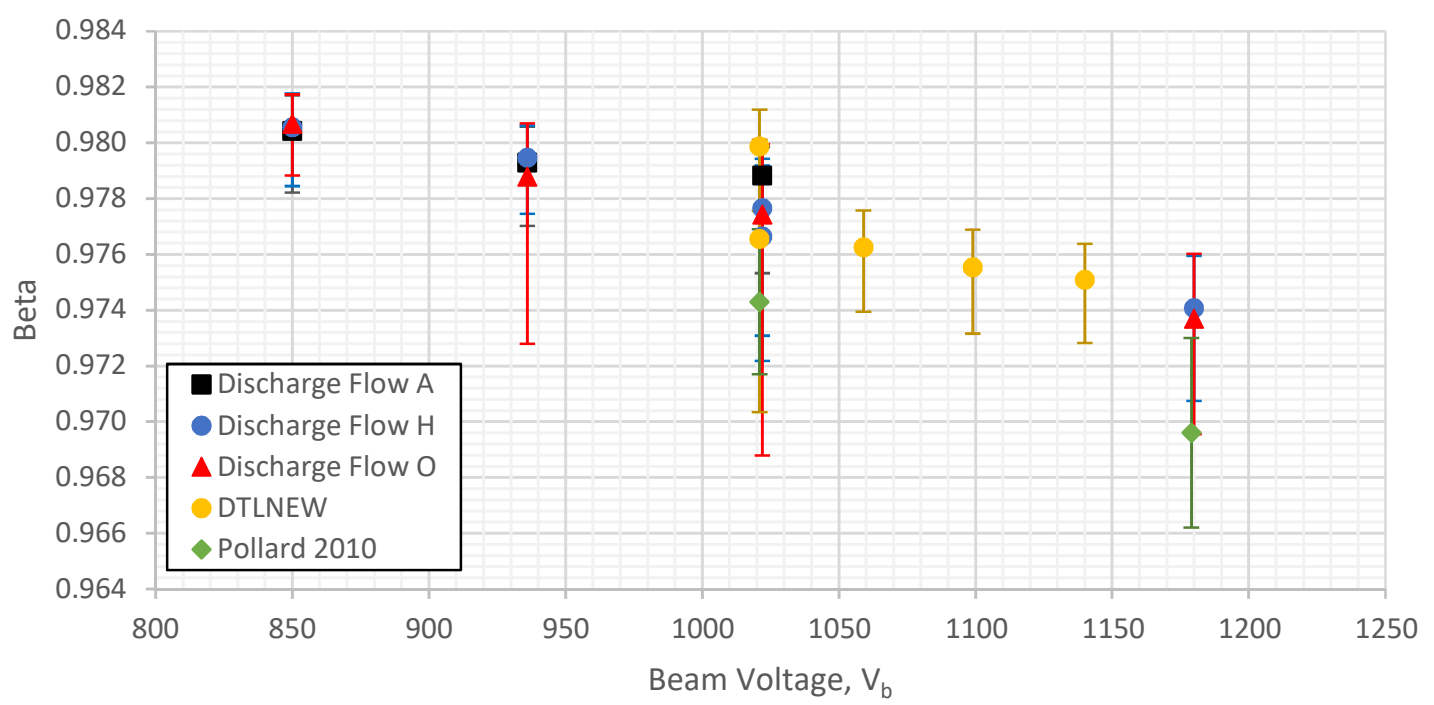

Figure 10 Divergence factor $\beta$ versus beam power supply voltage $V_{b}$.

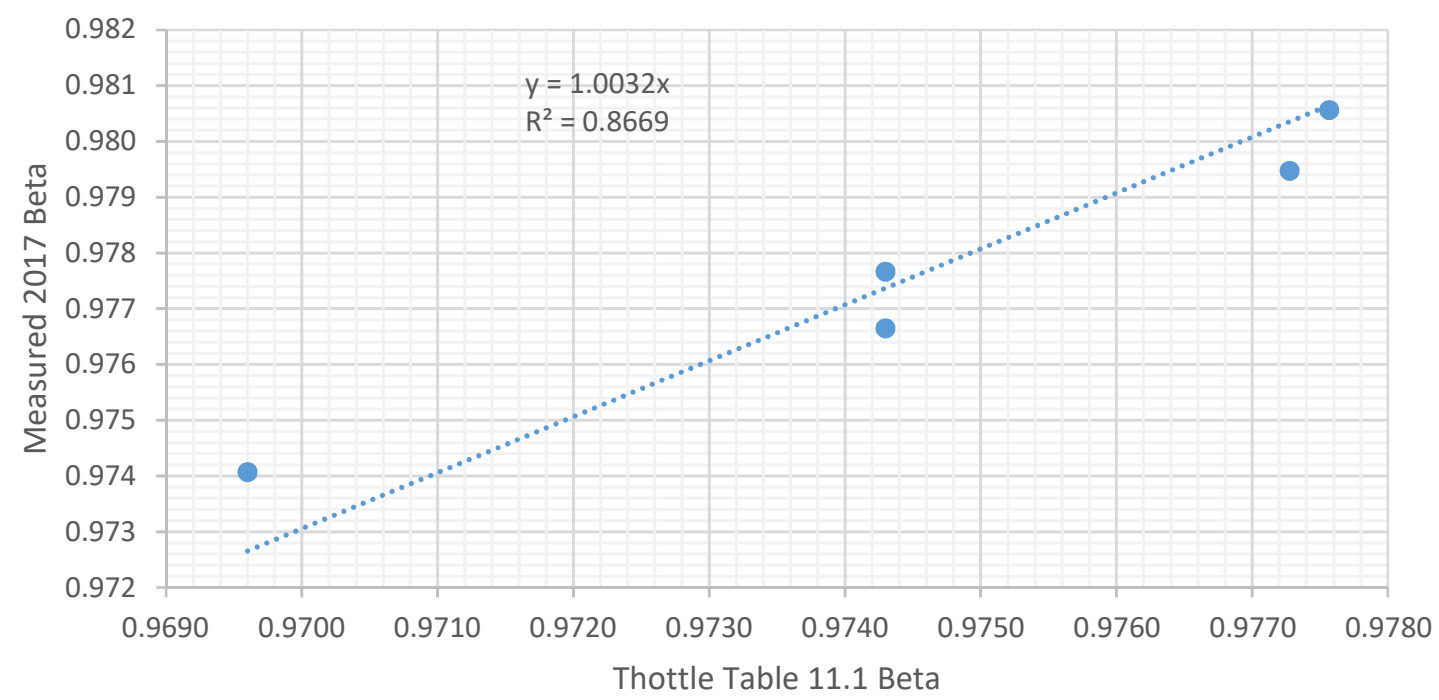

Figure 11 Measured versus predicted divergence factor $\beta$.

\section{Conclusions}

Overall, this study has shown that divergence measurements of the NEXT thruster, even with different thruster test artles, are highly repeatable. The current density distributions and associated divergence factors essentially do not vary with flow rate. Furthermore, at fairly short distances compared to the thruster dimension (about 4-5 diameters, similar to predicted in [15]), one can get reliable distribution functions that can be used to determine divergence with high precision. The key is using the correct assumptions about ion trajectories and making the appropriate correction to probe flux. At 1m, with the appropriate probe geometry, it appears one can reliably capture distance-independent, high angle current density distributions. These distributions seem to hint at a variety of interesting plume features, at increasing low current levels. In the past, these features have been attributed to low energy ions (e.g. [17]), however our RPA measurements indicate they have at least $35 \mathrm{eV}$ in energy, if not more. The physical origin and complete 
energy distribution of these peripheral ions may be worth further investigation. That said, the current density at high angles for energetic ions drops by over five decades from its peak value. In contrast, the charge exchange current density at $1 \mathrm{~m}$ appears to settle at baseline value by $45^{\circ}$ and remain at that value out to high angles.

\section{Acknowledgments}

The authors thank Kevin McCormick of NASA GRC for assistance in the setup and alignment of the thrust system and diagnostics and Rohit Shastry, also of NASA GRC, for technical discussions. We would also like to thank Mike Worshum, Phillip Mak, and Byron Zeigler of The Aerospace Corporation for technical support.

\section{References}

[1] Shastry R., Herman, D.A., Soulas, G.C., Patterson, M.J., "Status of NASA's Evolutionary Xenon Thruster (NEXT) LongDuration Test as of 50,000 h and 900 kg Throughput." Paper IEPC-2013-121, 33rd International Electric Propulsion Conf., Oct. 2013.

[2] Crofton M.W., Nocerino J.C., Young J.A., and Patterson M.J., "NEXT Ion Engine Plume Deposition Rates: QCM Measurements," AIAA Paper 2014-0140, SciTech 2014 Conference, National Harbor, MD, January 2014.

[3] Crofton M.W. and Patterson M.J., "Molybdenum LIF and NEXT Erosion Rate," Paper IEPC-2015-183/ISTS-2015-b-183, 34th International Electric Propulsion Conference, July 2015.

-[4] Patterson, M.J., Soulas, G.C., Young, J.A., and Crofton, M.W., "Expanded Throttling Capabilities of the NEXT Thruster," 49th Joint Propulsion Conference, July 2013.

[5] Young J. A., Crofton M.W., and Patterson M.J. "Plume Characterization of the NEXT Thruster Under Extended Throttle Conditions", 49th AIAA/ASME/SAE/ASEE Joint Propulsion Conference, Joint Propulsion Conferences, (AIAA 2013-4111)

-[6] Pollard, J.E., Diamant K.D., Crofton M.W., Patterson, M.J., Soulas, G.C., "Spatially-Resolved Beam Current and ChargeState Distributions for the NEXT Ion Engine," AIAA Paper No. 2010-6779, 46 "th Joint Propulsion Conference, July 2010.

[7] Diamant K.D., Pollard, J.E., Crofton M.W., Patterson, M.J., Soulas, G.C., "Thrust Stand Characterization of the NASA Evolutionary Xenon Thruster (NEXT)," AIAA Paper No. 2010-6701, 46 ${ }^{\text {th }}$ Joint Propulsion Conference, July 2010.

-[8] Patterson, M.J., "NEXT Study of Thruster Extended-Performance II (NEXT STEP II)," AIAA Paper No. 2008-4808, 44th Joint Propulsion Conference and Exhibit, Hartford, CT, July 2008.

-[9] Patterson, M.J., "NEXT Study of Thruster Extended Performance (NEXT STEP)", AIAA Paper No. 2006-4664, 42nd Joint Propulsion Conference and Exhibit, Sacramento, CA, July 2006.

[10] Sarli B.V., Ozimek M.T., Atchison J.A., Englander J.A.and Barbee B.W., "NASA Double Asteroid Redirection Test (DART) Trajectory Validation and Robustness." AAS Paper 17-206, 27th AAS/AIAA Space Flight Mechanics Meeting, San Antonio, TX, 5-9 Feb. 2017.

[11] Matlock, T.S. et al, “Thrust Vector Measurements for NEXT: DART Mission,” AIAA Science and Technology Forum, San Diego, January 2019.

[12] Crofton, M.W. et al, "LIF Erosion Rate Measurements of NEXT Ion Engine for DART Mission," AIAA Science and Technology Forum, San Diego, January 2019.

[13] Crofton, M.W. et al, "Deposition Rate Measurements in NEXT Ion Engine Plume for DART Mission," AIAA Science and Technology Forum, San Diego, January 2019.

-[14] Danilowicz R. L., Rawlin V.K., Banks B.A., and Wintucky E.G., "Measurement of Beam Divergence of 30-Centimeter Dished Grids", AIAA Paper 73-1051, 10 ${ }^{\text {th }}$ Electric Propulsion Conf., Oct. 1973.

-[15] Reynolds T.W., "Mathematical Representation of Current Density Profiles from Ion Thrusters" NASA TN D-6334, April 1971.

[16] Aston G., Kaufman H. R., and Wilbur P. J. "Ion beam divergence characteristics of two-grid accelerator systems", AIAA Journal, Vol. 16, No. 5 (1978), pp. 516-524.

-[17] Snyder J.S., et al. "Performance Evaluation of the T6 Ion Engine", AIAA Paper 2010-7114, 46th AIAA/ASME/SAE/ASEE Joint Propulsion Conference \& Exhibit, Joint Propulsion Conferences, July 2010.

-[18] Soulas G, Kamhawi H, Patterson M, Britton M, and Frandina M. "NEXT Ion Engine 2000 Hour Wear Test Results", AIAA Paper 2004-3791, 40th AIAA/ASME/SAE/ASEE Joint Propulsion Conf, Ft Lauderdale, FL, July 11-14, 2004

[19] Peng E, Yu D, Jiang B, "Experimental Investigation of Backpressure Effects on the Ionization and Acceleration Processes in a Hall Thruster" Paper IEPC-2009-119, 31 ${ }^{\text {st }}$ International Electric Propulsion Conf, Ann Arbor, MI, Sept, 20-24, 2009.

[20] Shastry R, Herman D.A., Soulas G.C., and Patterson M.J.. "End-of-test Performance and Wear Characterization of NASA's Evolutionary Xenon Thruster (NEXT) Long-Duration Test", AIAA Paper 2014-3617, 50th AIAA/ASME/SAE/ASEE Joint Propulsion Conf,, Cleveland, OH, July 28-30, 2014. 